MATHEMATICS OF COMPUTATION

Volume 76, Number 260, October 2007, Pages 1981-1993

S 0025-5718(07)01988-6

Article electronically published on May 9, 2007

\title{
ON GEOMETRIC INTERPOLATION BY PLANAR PARAMETRIC POLYNOMIAL CURVES
}

\author{
GAŠPER JAKLIČ, JERNEJ KOZAK, MARJETA KRAJNC, AND EMIL ŽAGAR
}

\begin{abstract}
In this paper the problem of geometric interpolation of planar data by parametric polynomial curves is revisited. The conjecture that a parametric polynomial curve of degree $\leq n$ can interpolate $2 n$ given points in $\mathbb{R}^{2}$ is confirmed for $n \leq 5$ under certain natural restrictions. This conclusion also implies the optimal asymptotic approximation order. More generally, the optimal order $2 n$ can be achieved as soon as the interpolating curve exists.
\end{abstract}

\section{INTRODUCTION}

Geometric interpolation by parametric polynomial curves has received considerable attention since it was introduced in [2]. Perhaps one of the reasons for this is the fact that the interpolating curve depends on parametrization-independent geometric quantities such as data points, tangent directions, curvatures, etc. This makes the geometric interpolant a valuable tool in the computer aided geometric design. Furthermore, it is well known that geometric interpolation schemes can provide interpolating curves of high accuracy. In 4 it has been conjectured that a parametric polynomial curve of degree $n$ in $\mathbb{R}^{d}$ can interpolate $n+1+\lfloor(n-1) /(d-1)\rfloor$ given data. There are only a few results for a particular choice of parameters $n$ and $d$. The most general result (but still not optimal) is [9], and it confirms that $n+1+\lfloor(n+1) /(2 d-1)\rfloor$ points can be interpolated at least asymptotically. Obviously this conjecture is particularly interesting in low dimensions, i.e., $d=2,3$. In the planar case it reduces to a guess that $2 n$ data values can be interpolated, and the approximation order $2 n$ achieved. Compared to the functional case this would be a much stronger result.

But unfortunately, geometric interpolating schemes are nonlinear. This drawback makes it hard to analyse the existence of the interpolating curve and to establish the approximation order. Numerical computations have to be done with some care too, usually by the continuation method (11). Thus it is quite clear why the analysis of geometric interpolation schemes is usually based upon the assumption that data are sampled densely enough from a smooth curve, and the asymptotic analysis is applied. Perhaps there is only one exception to the above approach, observed in [10, 7, 8, 3] and extended to the general $d$ in [5], i.e., the case $n=d$.

Received by the editor September 4, 2006 and, in revised form, September 7, 2006.

2000 Mathematics Subject Classification. Primary 41A05, 41A10, 41A25, 65D05, 65D17; Secondary 65D10.

Key words and phrases. Geometric interpolation, approximation order, asymptotic analysis.

The second and fourth authors were partially supported by Ministry of Higher Education, Science and Technology of Slovenia.

(C)2007 American Mathematical Society Reverts to public domain 28 years from publication 
In this paper geometric interpolation by planar parametric polynomial curves is studied, and the conjecture in [4] reconsidered. In section 2 the interpolation problem is formulated and the equations that determine the interpolating curve are derived. Sections 3 and 4 provide a general setup for the asymptotic analysis and the study of the approximation order. The last section outlines the asymptotic analysis for $n=5$. The main conclusions of the paper are: if the data, sampled from a convex smooth curve, are close enough, then

- equations that determine the interpolating polynomial curve are derived for general $n$ (Theorem 4.5),

- if the interpolating polynomial curve exists, the approximation order is $2 n$ for general $n$ (Theorem 4.6),

- the interpolating polynomial curve exists for $n \leq 5$ (Theorem 4.7).

In order to keep the paper technically as simple as possible, only Lagrange interpolation is discussed. However, section 4, in particular Theorem 4.5, reveals that the results of the asymptotic analysis can be carried over to the multiple geometric interpolation (interpolation of a point, a tangent direction at that point, a curvature at that point, etc.), as well as to the Taylor interpolation case considered in [8. 11.

\section{INTERPOLATION PROBLEM}

The interpolation problem is formulated as follows. Suppose that a sequence of $2 n$ distinct points $\boldsymbol{T}_{0}, \boldsymbol{T}_{1}, \ldots, \boldsymbol{T}_{2 n-1}$ in the plane $\mathbb{R}^{2}$ is given. Find a parametric polynomial curve

$$
\boldsymbol{P}_{n}:[0,1] \rightarrow \mathbb{R}^{2}
$$

of degree $\leq n$ that interpolates the given points at some values $t_{\ell} \in[0,1]$ in increasing order, i.e.,

$$
\boldsymbol{P}_{n}\left(t_{j}\right)=\boldsymbol{T}_{j}, \quad j=0,1, \ldots, 2 n-1 .
$$

Since a linear transformation of the parameter preserves the degree of a parametric polynomial curve, one can assume $t_{0}:=0$ and $t_{2 n-1}:=1$, but the remaining parameters

are unknown, ordered as

$$
\boldsymbol{t}:=\left(t_{\ell}\right)_{\ell=1}^{2 n-2}
$$

$$
t_{0}=0<t_{1}<\cdots<t_{2 n-2}<t_{2 n-1}=1 .
$$

The system of equations (2.1) should determine the unknown $\boldsymbol{P}_{n}$ as well as the parameters $\boldsymbol{t}$. But the two tasks can be separated if one can provide enough linearly independent functionals, depending on $\boldsymbol{t}$ only, that map $\boldsymbol{P}_{n}$ to zero. Divided differences, based upon $\geq n+2$ values, are a natural choice. Let us apply the divided differences

$$
\left[t_{j-1}, t_{j}, \ldots, t_{n+j}\right], \quad j=1,2, \ldots, n-1,
$$

to both sides of (2.1). Since $\operatorname{deg} P_{n} \leq n$, the left side vanishes, and so should the right one. But the $t_{\ell}$ are distinct and this condition becomes

$$
\sum_{\ell=j-1}^{n+j} \frac{\boldsymbol{T}_{\ell}}{\prod_{\substack{m=j-1 \\ m \neq \ell}}^{n+j}\left(t_{\ell}-t_{m}\right)}=0, \quad j=1,2, \ldots, n-1 .
$$


This nonlinear system depends on the data $\boldsymbol{T}_{\ell}$ and the unknowns $\boldsymbol{t}$ only. For each $j$ it provides two equations based upon the first and the second component of the data. The solution of the system (2.3) may or may not exist. The difficult part of the interpolation problem is to find it. If the unknowns $\boldsymbol{t}$ have already been determined, it is straightforward to obtain the polynomial curve $\boldsymbol{P}_{n}$. One only has to take any $n+1$ distinct interpolating conditions in (2.1), and apply any standard linear interpolation scheme to $\boldsymbol{P}_{n}$ componentwise.

\section{Asymptotic ApProACH}

It seems hard to analyse the nonlinear system of equations (2.3) without additional restrictions. Here, the asymptotic approach will be applied, with the assumption that the points $\boldsymbol{T}_{\ell}$ are sampled from a smooth regular convex planar parametric curve $f:[0, h] \rightarrow \mathbb{R}^{2}$. The length of the parameter interval $h$ is supposed to be small enough so that a local expansion of $f$ around 0 can be applied. Affine transformations of the points $\boldsymbol{T}_{\ell}$ transform (2.3) to an equivalent form. Thus one can assume $\boldsymbol{f}(0)=\left(\begin{array}{l}0 \\ 0\end{array}\right)$ and $\boldsymbol{f}^{\prime}(0)=\left(\begin{array}{l}1 \\ 0\end{array}\right)$, and parameterize $\boldsymbol{f}$ by the first component

$$
\boldsymbol{f}(x)=\left(\begin{array}{c}
x \\
y(x)
\end{array}\right)
$$

where $y$ expands as

$$
y(x)=\frac{1}{2} y^{\prime \prime}(0) x^{2}+\frac{1}{3 !} y^{(3)}(0) x^{3}+\cdots+\frac{1}{(2 n-1) !} y^{(2 n-1)}(0) x^{2 n-1}+\mathcal{O}\left(x^{2 n}\right) .
$$

The curve is assumed to be convex, which implies $y^{\prime \prime}(0)>0$. We will be looking for the values of $f$ at small values of $h$, therefore the coordinate system needs an appropriate scaling by the matrix

$$
D_{h}:=\operatorname{diag}\left(\frac{1}{h}, \frac{2}{y^{\prime \prime}(0) h^{2}}\right)
$$

Now let $\boldsymbol{T}_{\ell}$ be the points on the curve $\boldsymbol{f}$, taken at different parameter values in $[0, h]$. Then for some $\eta_{\ell}$,

$$
\eta_{0}:=0<\eta_{1}<\cdots<\eta_{2 n-2}<\eta_{2 n-1}:=1
$$

the data points are chosen as $\boldsymbol{T}_{\ell}=D_{h} \boldsymbol{f}\left(\eta_{\ell} h\right)$. Their expansion in $h$ is

$$
\boldsymbol{T}_{\ell}=\left(\begin{array}{c}
\eta_{\ell} \\
\sum_{k=2}^{\infty} c_{k} h^{k-2} \eta_{\ell}^{k}
\end{array}\right), \quad \ell=0,1, \ldots, 2 n-1
$$

Here, the constants $c_{k}$ depend on $y$, but not on $\eta_{\ell}$ or $h$, i.e.,

$$
c_{k}=\frac{2}{k !} \frac{y^{(k)}(0)}{y^{\prime \prime}(0)}, \quad k=2,3, \ldots
$$




\section{System of EQUations in ASYMPtotic FORM}

In this section the system (2.3) is analysed, where the data points are given by (3.3) and $h$ is small enough. As far as the existence of the solution is concerned, one has to show that there exists $h_{0}>0$, such that the system (2.3) has a solution $\boldsymbol{t}$ for all $h, 0 \leq h \leq h_{0}$. The solution is easy to guess at the limit value $h=0$, i.e.,

$$
\boldsymbol{t}=\boldsymbol{\eta}:=\left(\eta_{\ell}\right)_{\ell=1}^{2 n-2},
$$

since

$$
\lim _{h \rightarrow 0} \boldsymbol{T}_{\ell}=\left(\begin{array}{l}
\eta_{\ell} \\
\eta_{\ell}^{2}
\end{array}\right)
$$

and

$$
\left[\eta_{j-1}, \eta_{j}, \ldots, \eta_{n+j}\right]_{\eta}\left(\begin{array}{c}
\eta \\
\eta^{2}
\end{array}\right)=\left(\begin{array}{l}
0 \\
0
\end{array}\right), \quad j=1,2, \ldots, n-1 .
$$

In view of (4.1) it is important to study the unknown differences

$$
\eta_{\ell}-t_{\ell}, \quad \ell=1,2, \ldots, 2 n-2,
$$

as functions of $h$. It does not matter if (4.2) is studied with $\eta_{\ell}$ given and $t_{\ell}$ unknown or vice versa. From now on it will be simpler to assume that $\boldsymbol{t}$ are given parameters and $\boldsymbol{\eta}$ are the unknowns, as in [8]. Furthermore, the system of equations (2.3) will be rewritten in an equivalent form, with divided differences (2.2) replaced by their linear combinations, i.e.,

$$
\left[t_{0}, t_{1}, \ldots, t_{n+j}\right], \quad j=1,2, \ldots, n-1 .
$$

With the notation

$$
\omega_{j}(t):=\prod_{\ell=0}^{n+j}\left(t-t_{\ell}\right), \quad \dot{\omega}_{j}(t):=\frac{d \omega_{j}(t)}{d t}, \quad j=1,2, \ldots, n-1,
$$

the system (2.3) is transformed into

$$
\sum_{\ell=0}^{n+j} \frac{1}{\dot{\omega}_{j}\left(t_{\ell}\right)} \boldsymbol{T}_{\ell}=0, \quad j=1,2, \ldots, n-1 .
$$

Of course, the limit properties of the system are preserved, since the linear transformation from (2.2) to (4.3) is invertible.

Unfortunately, the implicit function theorem cannot be applied to extend the limit solution $\boldsymbol{\eta}=\boldsymbol{t}$ continuously to $h>0$ for $n>2$. This is obvious from the following theorem.

Theorem 4.1. Let $J$ be the Jacobian of the system (4.4) with respect to the unknowns $\boldsymbol{\eta}$ at $h=0$. Then

$$
\operatorname{dim} \operatorname{ker} J=n-2 .
$$

Proof. The Jacobian $J$ is easily computed from

$$
\left.\left(\frac{\partial}{\partial \eta_{m}} \boldsymbol{T}_{\ell}\right)\right|_{\boldsymbol{\eta}=\boldsymbol{t}, h=0}=\left(\begin{array}{c}
\delta_{\ell, m} \\
2 \delta_{\ell, m} t_{\ell}
\end{array}\right), \quad \ell, m=1,2, \ldots, 2 n-2,
$$

and from the system (4.4). Let

$$
\boldsymbol{x}_{i}^{T}:=(\underbrace{0, \ldots, 0}_{2 i-2},-2,0,-2 t_{n+1+i}, 1,0,0, \ldots, 0)^{T}, \quad i=1,2, \ldots, n-2 .
$$


Observe that

$$
\left(\boldsymbol{x}_{i}^{T} J\right)_{m}=\left\{\begin{array}{lll}
-\frac{2}{\dot{\omega}_{i}\left(t_{m}\right)}-\frac{2 t_{n+1+i}}{\dot{\omega}_{i+1}\left(t_{m}\right)}+\frac{2 t_{m}}{\dot{\omega}_{i+1}\left(t_{m}\right)} & =0, & m=1,2, \ldots, n+i, \\
-\frac{2 t_{n+1+i}}{\dot{\omega}_{i+1}\left(t_{m}\right)}+\frac{2 t_{n+1+i}}{\dot{\omega}_{i+1}\left(t_{m}\right)} & =0, & m=n+1+i, \\
0, & \text { otherwise. }
\end{array}\right.
$$

But the $\boldsymbol{x}_{i}^{T}$ are linearly independent, hence $\operatorname{dim} \operatorname{ker} J \geq n-2$. Let

$$
M:=J\left(\begin{array}{cccccc}
2 & 1 & 3 & 5 & \ldots & 2 n-3 \\
1 & 2 & 3 & 4 & \cdots & n
\end{array}\right) \cdot \operatorname{diag}\left(\dot{\omega}_{n-1}\left(t_{1}\right), \dot{\omega}_{n-1}\left(t_{2}\right), \ldots, \dot{\omega}_{n-1}\left(t_{n}\right)\right) .
$$

It is easy to see that $M=\left(\phi_{j}\left(t_{m}\right)\right)_{j=1, m=1}^{n, n}$, where the polynomials $\phi_{j}$ are given as

$$
\phi_{1}(t):=2 t \prod_{\ell=n+2}^{2 n-1}\left(t-t_{\ell}\right), \quad \phi_{j}(t)=\prod_{\ell=n+j}^{2 n-1}\left(t-t_{\ell}\right), \quad j=2,3, \ldots, n .
$$

This implies that $M$ must be nonsingular. If not, its rows would be linearly dependent and there would exist a polynomial $\sum_{j=1}^{n} \gamma_{j} \phi_{j}$ of degree $\leq n-1$ with $n$ roots $t_{m}, m=1,2, \ldots, n$, an obvious contradiction. So rank $J \geq n$, and the result of the lemma follows.

Thus a more refined existence analysis has to be applied. The system of equations (4.4) will now be split in two parts, the equations determined by the first components of the points $\boldsymbol{T}_{\ell}$, and the equations provided by the second ones. A proper reparametrization of the curve $\boldsymbol{f}$, the idea heavily leaned upon in [8], will yield a simple solution of the first part. Let us introduce new unknowns $\boldsymbol{\xi}:=\left(\xi_{\ell}\right)_{\ell=1}^{2 n-2}$ by a reparametrization of the curve $\boldsymbol{f}$ as

$$
\eta \rightarrow \eta(t):=\eta(t ; \boldsymbol{\xi})
$$

given at $t_{\ell}$ as

$$
\eta_{\ell}=\eta\left(t_{\ell} ; \boldsymbol{\xi}\right)=t_{\ell}+u\left(t_{\ell} ; \boldsymbol{\xi}\right)+\xi_{n-2+\ell} h^{n-1} p\left(t_{\ell}\right), \quad \ell=1,2, \ldots, 2 n-2,
$$

where $\xi_{\ell}:=0, \ell>2 n-2$. Furthermore, let

$$
p(t):=\left(t-t_{0}\right) \prod_{\ell=n+1}^{2 n-1}\left(t-t_{\ell}\right), \quad u(t ; \boldsymbol{\xi}):=\left(t-t_{0}\right)\left(t-t_{2 n-1}\right) \sum_{j=1}^{n-2} \xi_{j} h^{j} t^{j-1} .
$$

The reparametrization (4.6) is quite clearly regular for $\boldsymbol{\xi}$ bounded independently of $h$ and $h$ small enough, since

$$
\eta\left(t_{0} ; \boldsymbol{\xi}\right)=t_{0}=0=\eta_{0}, \quad \eta\left(t_{2 n-1} ; \boldsymbol{\xi}\right)=t_{2 n-1}=1=\eta_{2 n-1}, \quad \eta^{\prime}(t ; \boldsymbol{\xi})=1+\mathcal{O}(h) .
$$

The limit conditions $\eta_{\ell}=t_{\ell}$ at $h=0$ are fulfilled too.

Lemma 4.2. The change of variables $\boldsymbol{\eta} \rightarrow \boldsymbol{\xi}$ introduced in (4.7) is one-to-one.

Proof. Note that $p\left(t_{\ell}\right)=0, \ell=n+1, n+2, \ldots, 2 n-2$. So (4.7) provides the conditions $\eta_{\ell}=t_{\ell}+u\left(t_{\ell} ; \boldsymbol{\xi}\right), \ell=n+1, n+2, \ldots, 2 n-2$, that uniquely determine the polynomial

$$
\sum_{j=1}^{n-2} \xi_{j} h^{j} t^{j-1}
$$

of degree $<n-2$ since $t_{\ell}$ are distinct. But then the rest of the new unknowns $\left(\xi_{\ell}\right)_{\ell=n-1}^{2 n-2}$ are obtained from (4.7) by choosing $\ell=1,2, \ldots, n$. 
Let $\boldsymbol{T}_{\ell}$ be given by (3.3), with the reparametrization (4.7) applied. The system (4.4) can now be written as

$$
\begin{aligned}
& \boldsymbol{F}(\boldsymbol{\xi} ; h):=\left(F_{j}(\boldsymbol{\xi} ; h)\right)_{j=1}^{n-1}=\mathbf{0}, \\
& \boldsymbol{G}(\boldsymbol{\xi} ; h):=\left(G_{j}(\boldsymbol{\xi} ; h)\right)_{j=1}^{n-1}=\mathbf{0},
\end{aligned}
$$

where

$$
F_{j}(\boldsymbol{\xi} ; h):=\sum_{\ell=0}^{n+j} \frac{1}{\dot{\omega}_{j}\left(t_{\ell}\right)}\left(t_{\ell}+u\left(t_{\ell} ; \boldsymbol{\xi}\right)+\xi_{n-2+\ell} h^{n-1} p\left(t_{\ell}\right)\right)
$$

and

$$
G_{j}(\boldsymbol{\xi} ; h):=\sum_{\ell=0}^{n+j} \frac{1}{\dot{\omega}_{j}\left(t_{\ell}\right)}\left(\sum_{k=2}^{\infty} c_{k} h^{k-2}\left(t_{\ell}+u\left(t_{\ell} ; \boldsymbol{\xi}\right)+\xi_{n-2+\ell} h^{n-1} p\left(t_{\ell}\right)\right)^{k}\right) .
$$

The following result has been conjectured from some numerical experiments.

Theorem 4.3. The unknowns $\boldsymbol{\xi}$ can solve (4.9) if and only if

$$
\xi_{n-1}=\xi_{n}=\xi_{n+1}=\cdots=\xi_{2 n-2} .
$$

Proof. A divided difference is a linear functional, so the functions $F_{j}$, defined in (4.10), can be simplified to

$$
\begin{aligned}
F_{j}(\boldsymbol{\xi} ; h) & =\left[t_{0}, t_{1}, \ldots, t_{n+j}\right]_{t}(t+u(t ; \boldsymbol{\xi}))+h^{n-1} \sum_{\ell=0}^{n+j} \frac{1}{\dot{\omega}_{j}\left(t_{\ell}\right)} \xi_{n-2+\ell} p\left(t_{\ell}\right) \\
& =h^{n-1} \sum_{\ell=0}^{n+j} \frac{1}{\dot{\omega}_{j}\left(t_{\ell}\right)} \xi_{n-2+\ell} p\left(t_{\ell}\right),
\end{aligned}
$$

since the polynomial $t+u(t ; \boldsymbol{\xi})$ is of degree $\leq n-1$ in $t$. Further, the polynomial $p$ is of the particular form (4.8) and $\operatorname{deg} p=n$, so it follows that (4.10) reads

$$
\begin{aligned}
0=\sum_{\ell=0}^{n+j} \frac{1}{\dot{\omega}_{j}\left(t_{\ell}\right)} \xi_{n-2+\ell} p\left(t_{\ell}\right) & =\sum_{\ell=0}^{n+j} \frac{1}{\dot{\omega}_{j}\left(t_{\ell}\right)} \xi_{n-2+\ell} p\left(t_{\ell}\right)-\xi_{n-1} \sum_{\ell=0}^{n+j} \frac{1}{\dot{\omega}_{j}\left(t_{\ell}\right)} p\left(t_{\ell}\right) \\
& =\sum_{\ell=0}^{n+j} \frac{1}{\dot{\omega}_{j}\left(t_{\ell}\right)}\left(\xi_{n-2+\ell}-\xi_{n-1}\right) p\left(t_{\ell}\right) \\
& =\sum_{\ell=2}^{n} \frac{1}{\dot{\omega}_{j}\left(t_{\ell}\right)}\left(\xi_{n-2+\ell}-\xi_{n-1}\right) p\left(t_{\ell}\right) .
\end{aligned}
$$

It is easy to verify that the square matrix

$$
A:=\left(\frac{1}{\dot{\omega}_{j}\left(t_{\ell}\right)}\right)_{j=1 ; \ell=2}^{n-1 ; n}
$$

is nonsingular by finding a closed form of $\operatorname{det} A$ (see [6], e.g.). So it can map only the trivial vector to $\mathbf{0}$. Since $p\left(t_{\ell}\right) \neq 0, \ell=2,3, \ldots, n$, the term $\xi_{n-2+\ell}-\xi_{n-1}$ should vanish for all $\ell$ concerned, and the claim (4.11) follows.

Theorem 4.3 pins down the choice $\xi_{j}=\xi_{n-1}, j=n, n+1, \ldots, 2 n-2$, that will be assumed from now on. The rest of the unknowns $\left(\xi_{\ell}\right)_{\ell=1}^{n-1}$ should be determined 
by the second part of equations (4.9). But (4.11) simplifies the reparametrization (4.6) to a polynomial

$$
\eta(t ; \boldsymbol{\xi})=t+u(t ; \boldsymbol{\xi})+\xi_{n-1} h^{n-1} p(t)
$$

and further $G_{j}(\boldsymbol{\xi} ; h)$ to

$$
G_{j}(\boldsymbol{\xi} ; h)=\left[t_{0}, t_{1}, \ldots, t_{n+j}\right] \sum_{k=2}^{\infty} c_{k} h^{k-2} \eta(\cdot ; \boldsymbol{\xi})^{k} .
$$

In order to study (4.14) further the following lemma is needed.

Lemma 4.4. Let

$$
q(t ; \boldsymbol{\xi}):=t+\sum_{\ell=1}^{n-1} \xi_{\ell} h^{\ell} t^{\ell+1}
$$

Then

$$
\left[t_{0}, t_{1}, \ldots, t_{n+j}\right] \eta(\cdot ; \boldsymbol{\xi})^{k}=\left[t_{0}, t_{1}, \ldots, t_{n+j}\right] q(\cdot ; \boldsymbol{\xi})^{k}+\mathcal{O}\left(h^{n+j+1-k}\right)
$$

and

$$
\left[t_{0}, t_{1}, \ldots, t_{n+j}\right] q(\cdot ; \boldsymbol{\xi})^{k}=\mathcal{O}\left(h^{n+j-k}\right) .
$$

Proof. Let us introduce some new notation. If $r$ is a polynomial in variables $t$ and $h$, then let

$$
\operatorname{termdeg}_{t}(r) \leq \operatorname{termdeg}_{h}(r)
$$

denote that for every term $t^{\alpha_{i}} h^{\beta_{i}}$ of $r$, the exponents $\alpha_{i}$ and $\beta_{i}$ satisfy the relation $\alpha_{i} \leq \beta_{i}$.

From (4.8) it is straightforward to verify that

$$
\operatorname{termdeg}_{t}(\eta) \leq \operatorname{termdeg}_{h}(\eta)+1,
$$

where $\eta$ is given by (4.13). Furthermore, the difference $\eta-q$ turns out as

$$
\begin{aligned}
\eta(t ; \boldsymbol{\xi})-q(t ; \boldsymbol{\xi})= & \left(-\left(t_{0}+t_{2 n-1}\right) t+t_{0} t_{2 n-1}\right) \sum_{j=1}^{n-2} \xi_{j} h^{j} t^{j-1} \\
& +\xi_{n-1} h^{n-1}\left(\left(t-t_{0}\right) \prod_{\ell=n+1}^{2 n-1}\left(t-t_{\ell}\right)-t^{n}\right),
\end{aligned}
$$

and clearly termdeg ${ }_{t}(\eta-q) \leq \operatorname{termdeg}_{h}(\eta-q)$. But

$$
\eta(t ; \boldsymbol{\xi})^{k}=q(t ; \boldsymbol{\xi})^{k}+\sum_{j=1}^{k}\left(\begin{array}{c}
k \\
j
\end{array}\right)(\eta(t ; \boldsymbol{\xi})-q(t ; \boldsymbol{\xi}))^{j} q(t ; \boldsymbol{\xi})^{k-j},
$$

and $q(t ; \boldsymbol{\xi})^{k}$ satisfies

$$
\operatorname{termdeg}_{t}\left(q^{k}\right)=\operatorname{termdeg}_{h}\left(q^{k}\right)+k \text {. }
$$

On the other hand, a brief look at the remaining sum yields

$$
\text { termdeg }_{t}\left(\eta^{k}-q^{k}\right) \leq \operatorname{termdeg}_{h}\left(\eta^{k}-q^{k}\right)+k-1 \text {. }
$$

The divided difference $\left[t_{0}, t_{1}, \ldots, t_{n+j}\right]_{t}$ maps polynomials in $t$ of degree $<n+j$ to zero. So the monomials with degree $=n+j$ will provide the leading term of the error. But then (4.16) and (4.17) confirm the lemma. 
Lemma 4.4 simplifies the functions (4.14) to

$$
G_{j}(\boldsymbol{\xi} ; h)=\left[t_{0}, t_{1}, \ldots, t_{n+j}\right] \sum_{k=2}^{\infty} c_{k} h^{k-2} q(\cdot ; \boldsymbol{\xi})^{k}+\mathcal{O}\left(h^{n+j-1}\right)
$$

and the following conclusion provides the final form of the system (4.9).

Theorem 4.5. The expansion referenced in (4.18) could be rewritten as

$$
\sum_{k=2}^{\infty} c_{k} h^{k-2} q(t ; \boldsymbol{\xi})^{k}=\sum_{k=2}^{\infty} C_{k}(\boldsymbol{\xi}) h^{k-2} t^{k}
$$

where

$$
C_{k}(\boldsymbol{\xi}):=\left.\frac{2}{k ! h^{k} y^{\prime \prime}(0)}\left(\frac{d^{k}}{d x^{k}} y(h q(x ; \boldsymbol{\xi}))\right)\right|_{x=0} .
$$

The polynomials $C_{k}(\boldsymbol{\xi})$ depend on $\boldsymbol{\xi}$ only, but not on $h$ or the parameters $\boldsymbol{t}$. So the final form of the system (4.9), for $h$ small enough, is given as

$$
C_{n+j}(\boldsymbol{\xi})+\mathcal{O}(h)=0, \quad j=1,2, \ldots, n-1 .
$$

Proof. Let us recall the proof of Lemma 4.4 and the notation (4.15). A close look reveals that

$$
\text { termdeg }{ }_{t}\left(h^{k-2} q^{k}\right)=\operatorname{termdeg}_{h}\left(h^{k-2} q^{k}\right)+2,
$$

hence (4.19) follows. But then

$$
\frac{1}{h^{n+j-2}} G_{j}(\boldsymbol{\xi} ; h)=C_{n+j}(\boldsymbol{\xi})+\mathcal{O}(h)=0, \quad j=1,2, \ldots, n-1 .
$$

Let us sum up all the asymptotic conclusions.

Theorem 4.6. If there exists $h_{0}>0$, such that the system of nonlinear equations (4.20) has a real solution for all $h, 0 \leq h \leq h_{0}$, then the interpolating polynomial parametric curve $\boldsymbol{P}_{n}$ exists and approximates $\boldsymbol{f}$ with the optimal approximation order, i.e., $2 n$.

Proof. The proof will follow the path already applied in [2]. If the interpolating curve $\boldsymbol{P}_{n}$ is reparametrized by a regular reparametrization $\varphi:[0, h] \rightarrow[0,1]$ in such a way that

$$
\left(\boldsymbol{P}_{n} \circ \varphi\right)\left(h \eta_{\ell}\right)=\boldsymbol{f}\left(h \eta_{\ell}\right), \quad \ell=0,1, \ldots, 2 n-1,
$$

the error analysis can be applied to each component separately, using the standard approach for the function case. But this implies that the optimal approximation order $2 n$ is achieved, provided $\left\|\left(\boldsymbol{P}_{n} \circ \varphi\right)^{(2 n)}\right\|$ remains bounded for all $h$ small enough.

By assumption the system (4.20) has a real solution and the unknown parameters $\boldsymbol{t}$ exist. Thus one can represent the curve $\boldsymbol{P}_{n}$ in the Lagrange form,

$$
\boldsymbol{P}_{n}=\left(\begin{array}{l}
P_{1} \\
P_{2}
\end{array}\right)=\sum_{\ell=0}^{n} \boldsymbol{f}\left(h \eta_{\ell}\right) \mathcal{L}_{\ell}, \quad \mathcal{L}_{\ell}(t):=\prod_{\substack{j=0 \\
j \neq \ell}}^{n} \frac{t-t_{j}}{t_{\ell}-t_{j}} .
$$

For the particular coordinate system, chosen in section 3 , a reparametrization $\varphi:=$ $P_{1}^{-1}$ is a proper choice. Indeed, from (4.22) and (4.7) it follows that

$$
P_{1}(t)=\sum_{\ell=0}^{n}\left(h \eta_{\ell}\right) \mathcal{L}_{\ell}(t)=h \sum_{\ell=0}^{n} \eta\left(t_{\ell} ; \boldsymbol{\xi}\right) \mathcal{L}_{\ell}(t)=h \eta(t ; \boldsymbol{\xi}),
$$


since the polynomial $\eta(\cdot ; \boldsymbol{\xi})$, defined in (4.13), is of degree $\leq n$. Note that

$$
P_{1}(0)=h \eta(0 ; \boldsymbol{\xi})=0, \quad P_{1}(1)=h \eta(1 ; \boldsymbol{\xi})=h, \quad P_{1}(t)=h t+\mathcal{O}\left(h^{2}\right) .
$$

So $P_{1}$ is a diffeomorphism $[0,1] \rightarrow[0, h]$ for $h$ small enough. The interpolation conditions (4.21) are satisfied, since

$$
\left(\boldsymbol{P}_{n} \circ P_{1}^{-1}\right)\left(h \eta_{\ell}\right)=\boldsymbol{P}_{n}\left(t_{\ell}\right)=\boldsymbol{f}\left(h \eta_{\ell}\right), \quad \ell=0,1, \ldots, 2 n-1,
$$

and $\varphi:=P_{1}^{-1}$ is the required reparametrization. In order to prove the boundedness of $\left(\boldsymbol{P}_{n} \circ \varphi\right)^{(2 n)}$ we apply the chain rule derivation to $\boldsymbol{P}_{n} \circ P_{1}^{-1}$. As already observed in [2] for the cubic case, and in [3] for general $n$, it suffices to see that $P_{1}^{\prime}(t)=$ $c h+\mathcal{O}\left(h^{2}\right), c \neq 0$, and

$$
P_{i}^{(k)}(t)=\mathcal{O}\left(h^{k}\right), \quad i=1,2, \quad k=2,3, \ldots, 2 n .
$$

Obviously $P_{1}^{\prime}(t)=h+\mathcal{O}\left(h^{2}\right)$. Since $\operatorname{deg} P_{i} \leq n$, it is enough to consider $2 \leq k \leq n$ only. The case $i=1$ follows immediately from (4.13) and (4.23). As to the other,

$$
P_{2}(t)=\sum_{\ell=0}^{n} y\left(h \eta_{\ell}\right) \mathcal{L}_{\ell}(t)=\sum_{\ell=0}^{n} y\left(h \eta\left(t_{\ell} ; \boldsymbol{\xi}\right)\right) \mathcal{L}_{\ell}(t) .
$$

Let us recall the expansion (3.1) from which we observe that the sums involved are

$$
\sum_{\ell=0}^{n}\left(h \eta\left(t_{\ell} ; \boldsymbol{\xi}\right)\right)^{m} \mathcal{L}_{\ell}(t)=\sum_{\ell=0}^{n} h^{m} t_{\ell}^{m} \mathcal{L}_{\ell}(t)+\mathcal{O}\left(h^{m+1}\right), \quad m \geq 2 .
$$

Since the interpolation is a projection on the space of polynomials of degree $\leq n$, the proof is complete.

Theorem 4.7. The system of nonlinear equations (4.20) has a real solution for $n \leq 5$, and $h$ small enough.

Proof. If $n=2$, the system (4.20) simplifies to one linear equation for $\xi_{1}$,

$$
2 \xi_{1}+c_{3}+\mathcal{O}(h)=0,
$$

which obviously has a real solution. The case $n=3$ is easy to analyse too, since the nonlinear system in this case is

$$
\begin{aligned}
\xi_{1}^{2}+3 c_{3} \xi_{1}+2 \xi_{2}+c_{4}+\mathcal{O}(h) & =0, \\
3 c_{3} \xi_{1}^{2}+2 \xi_{1}\left(\xi_{2}+2 c_{4}\right)+3 c_{3} \xi_{2}+c_{5}+\mathcal{O}(h) & =0 .
\end{aligned}
$$

But the first equation is always linear in $\xi_{n-1}=\xi_{2}$. So (4.24) can be reduced to a cubic equation for $\xi_{1}$,

$$
\xi_{1}^{3}+\frac{3}{2} c_{3} \xi_{1}^{2}+\left(\frac{9}{2} c_{3}^{2}-3 c_{4}\right) \xi_{1}+\frac{3}{2} c_{3} c_{4}-c_{5}+\mathcal{O}(h)=0,
$$

and the conclusion follows. The case $n=4$ will be omitted since the proof is, in the first part, very similar to that given in [11 and the other part is technically quite complicated. The proof for the case $n=5$ will be given in the following section. 


\section{THE CASE $n=5$}

Let us now consider the case $n=5$. After elimination of the variable $\xi_{4}=\xi_{n-1}$ the system (4.20) becomes

$$
P_{i}\left(\xi_{1}, \xi_{2}, \xi_{3}\right)+\mathcal{O}(h)=0, \quad i=1,2,3,
$$

with

$$
\begin{aligned}
& P_{1}\left(\xi_{1}, \xi_{2}, \xi_{3}\right) \\
& \quad:=-c_{3} \xi_{1}^{4}-\left(\frac{3}{2} c_{3}^{2}+2 c_{4}\right) \xi_{1}^{3}-\left(9 c_{3} c_{4}-5 c_{5}\right) \xi_{1}^{2}-\left(\frac{15}{2} c_{3} c_{5}-5 c_{6}\right) \xi_{1} \\
& \quad+\left(\frac{3}{2} c_{3}-\xi_{1}\right) \xi_{2}^{2}+\left(6 c_{3} c_{4}-5 c_{5}+\left(9 c_{3}^{2}-8 c_{4}\right) \xi_{1}-3 c_{3} \xi_{1}^{2}\right) \xi_{2} \\
& \quad-\left(\frac{9}{2} c_{3}^{2}-4 c_{4}+2 \xi_{1}^{2}-2 \xi_{2}\right) \xi_{3}-\frac{3}{2} c_{3} c_{6}+c_{7}, \\
& P_{2}\left(\xi_{1}, \xi_{2}, \xi_{3}\right) \\
& \quad:=-\left(3 c_{3}^{2}-c_{4}\right) \xi_{1}^{4}-\left(20 c_{3} c_{4}-10 c_{5}\right) \xi_{1}^{3}-\left(12 c_{4}^{2}+15 c_{3} c_{5}-15 c_{6}\right) \xi_{1}^{2} \\
& \quad-\left(10 c_{4} c_{5}+3 c_{3} c_{6}-7 c_{7}\right) \xi_{1}-2 c_{4} c_{6}+c_{8}-\xi_{2}^{3}-6 c_{3} \xi_{1} \xi_{2}^{2} \\
& \quad-\left(8 c_{4}^{2}-5 c_{6}+\left(24 c_{3} c_{4}-15 c_{5}\right) \xi_{1}+\left(18 c_{3}^{2}-6 c_{4}\right) \xi_{1}^{2}-c_{3} \xi_{1}^{3}\right) \xi_{2} \\
& \quad+\xi_{3}^{2}-\left(6 c_{3} c_{4}-5 c_{5}+\left(9 c_{3}^{2}-8 c_{4}\right) \xi_{1}+3 c_{3} \xi_{1}^{2}-\left(3 c_{3}-2 \xi_{1}\right) \xi_{2}\right) \xi_{3}, \\
& P_{3}\left(\xi_{1}, \xi_{2}, \xi_{3}\right) \\
& \quad:=-\frac{3}{2} c_{3}^{2} \xi_{1}^{5}-\left(15 c_{3} c_{4}-5 c_{5}\right) \xi_{1}^{4}-\left(36 c_{4}^{2}+10 c_{3} c_{5}-20 c_{6}\right) \xi_{1}^{3} \\
& \quad-\left(45 c_{4} c_{5}+\frac{3}{2} c_{3} c_{6}-21 c_{7}\right) \xi_{1}^{2}-\left(\frac{25}{2} c_{5}^{2}+6 c_{4} c_{6}-8 c_{8}\right) \xi_{1}-\frac{5}{2} c_{5} c_{6}+c_{9} \\
& \quad-2 c_{3} \xi_{2}^{3}-\left(12 c_{3} c_{4}-\frac{15}{2} c_{5}+\left(18 c_{3}^{2}-6 c_{4}\right) \xi_{1}+\frac{3}{2} c_{3} \xi_{1}^{2}\right) \xi_{2}^{2} \\
& \quad-\left(10 c_{4} c_{5}+3 c_{3} c_{6}-7 c_{7}+\left(24 c_{4}^{2}+30 c_{3} c_{5}-30 c_{6}\right) \xi_{1}\right. \\
& \left.\quad-\left(60 c_{3} c_{4}-30 c_{5}\right) \xi_{1}^{2}+\left(12 c_{3}^{2}-4 c_{4}\right) \xi_{1}^{3}\right) \xi_{2} \\
& \quad-2 \xi_{1} \xi_{3}^{2}-\left(\frac{15}{2} c_{3} c_{5}-5 c_{6}+\left(18 c_{3} c_{4}-10 c_{5}\right) \xi_{1}+\left(\frac{9}{2} c_{3}^{2}+6 c_{4}\right) \xi_{1}^{2}\right. \\
& \left.\quad-4 c_{3} \xi_{1}^{3}-\left(9 c_{3}^{2}-8 c_{4}+6 c_{3} \xi_{1}\right) \xi_{2}-\xi_{2}^{2}\right) \xi_{3} .
\end{aligned}
$$

The terms $\mathcal{O}(h)$ in (5.1) will be neglected for the moment. Let $\mathbb{R}\left[\xi_{1}, \xi_{2}, \ldots, \xi_{i}\right]$ denote the ring of polynomials in variables $\xi_{1}, \xi_{2}, \ldots, \xi_{i}$ over $\mathbb{R}$. A straightforward approach to the system (5.1) is right at hand: compute the Gröbner basis of the ideal

$$
\mathcal{I}:=\left\langle P_{1}, P_{2}, P_{3}\right\rangle \subset \mathbb{R}\left[\xi_{1}, \xi_{2}, \xi_{3}\right],
$$

and study the properties of the zeros of this basis, i.e., the variety $\mathcal{V}(\mathcal{I})$, the set of common zeros of $P_{i}, i=1,2,3$. But this approach is computationally too complex, and some ad hoc simplification is needed. The key conclusion is summarized in the following lemma.

Lemma 5.1. Let $\mathcal{I}$ be the ideal given in (5.2) and let $\mathcal{I}_{2}:=\mathcal{I} \cap \mathbb{R}\left[\xi_{1}\right]$ denote the second elimination ideal, obtained from $\mathcal{I}$ after elimination of $\xi_{3}$ and $\xi_{2}$. Then $\mathcal{V}\left(\mathcal{I}_{2}\right)=\mathcal{V}(Q)$ where $Q$ is a polynomial of degree $\leq 25$ in $\xi_{1}$, given as

$$
\begin{aligned}
Q\left(\xi_{1}\right)= & \frac{14641}{65536}\left(5 c_{3}^{2}-4 c_{4}\right)^{5} \xi_{1}^{25} \\
& -\frac{3025}{131072}\left(5 c_{3}^{2}-4 c_{4}\right)^{4}\left(1043 c_{3}^{3}-1988 c_{3} c_{4}+824 c_{5}\right) \xi_{1}^{24}+\ldots
\end{aligned}
$$


Proof. Consider the system (5.1). The first equation is linear in $\xi_{3}$, and can be written as

$$
P_{1}\left(\xi_{1}, \xi_{2}, \xi_{3}\right)=\psi_{1}\left(\xi_{1}, \xi_{2}\right)+\psi_{2}\left(\xi_{1}, \xi_{2}\right) \xi_{3}=0, \quad \psi_{1}, \psi_{2} \in \mathbb{R}\left[\xi_{1}, \xi_{2}\right]
$$

Similarly, the modified third equation

$$
P_{3}\left(\xi_{1}, \xi_{2}, \xi_{3}\right)+2 \xi_{1} P_{2}\left(\xi_{1}, \xi_{2}, \xi_{3}\right)=\chi_{1}\left(\xi_{1}, \xi_{2}\right)+\chi_{2}\left(\xi_{1}, \xi_{2}\right) \xi_{3}=0,
$$

with $\chi_{1}, \chi_{2} \in \mathbb{R}\left[\xi_{1}, \xi_{2}\right]$, turns out to be linear in $\xi_{3}$ too. One can now use the equation (5.3) to eliminate $\xi_{3}$, and the system (5.1) becomes

$$
\begin{aligned}
P_{12}\left(\xi_{1}, \xi_{2}\right):=\psi_{2}\left(\xi_{1}, \xi_{2}\right)^{2} P_{2}\left(\xi_{1}, \xi_{2},-\frac{\psi_{1}\left(\xi_{1}, \xi_{2}\right)}{\psi_{2}\left(\xi_{1}, \xi_{2}\right)}\right) & =0, \\
P_{13}\left(\xi_{1}, \xi_{2}\right):=\chi_{1}\left(\xi_{1}, \xi_{2}\right) \psi_{2}\left(\xi_{1}, \xi_{2}\right)-\chi_{2}\left(\xi_{1}, \xi_{2}\right) \psi_{1}\left(\xi_{1}, \xi_{2}\right) & =0 .
\end{aligned}
$$

Finally, the resultant of $P_{12}$ and $P_{13}$ with respect to $\xi_{2}$ is a single equation

$$
R_{1}\left(\xi_{1}\right):=\operatorname{Res}\left(P_{12}\left(\xi_{1}, \xi_{2}\right), P_{13}\left(\xi_{1}, \xi_{2}\right) ; \xi_{2}\right)=0 .
$$

The variety $\mathcal{V}\left(R_{1}\right) \supseteq \mathcal{V}\left(\mathcal{I}_{2}\right)$ may include some extraneous zeros introduced by the factor $\psi_{1}\left(\xi_{1}, \xi_{2}\right)^{2}$ in (5.5) or by the resultant Res. Also, the variety $\mathcal{V}$ does not precisely keep track of the multiple zeros, and the number of zeros of $R_{1}$ counting multiplicities could be bigger than the number of elements in $\mathcal{V}\left(R_{1}\right)$, i.e., $\# \mathcal{V}\left(R_{1}\right)$. The elimination procedure described also provides the extension path: if $\xi_{1} \in \mathcal{V}\left(I_{2}\right)$, the equations (5.5) determine $\xi_{2}$, and (5.3) finally $\xi_{3}$, except when $\psi_{2}\left(\xi_{1}, \xi_{2}\right)=0$, since then (5.3) leaves $\xi_{3}$ undefined. But then, at the first elimination step, one may choose the equation (5.4) rather than (5.3) to eliminate $\xi_{3}$. The equation (5.5) would be replaced by

$$
P_{32}\left(\xi_{1}, \xi_{2}\right):=\chi_{2}\left(\xi_{1}, \xi_{2}\right)^{2} P_{2}\left(\xi_{1}, \xi_{2},-\frac{\chi_{1}\left(\xi_{1}, \xi_{2}\right)}{\chi_{2}\left(\xi_{1}, \xi_{2}\right)}\right)=0,
$$

and one would finally be left with

$$
R_{2}\left(\xi_{1}\right):=\operatorname{Res}\left(P_{32}\left(\xi_{1}, \xi_{2}\right), P_{13}\left(\xi_{1}, \xi_{2}\right) ; \xi_{2}\right)=0 .
$$

Thus any $\xi_{1} \in \mathcal{V}\left(R_{1}\right) \cap \mathcal{V}\left(R_{2}\right)$ that is not extraneous can be extended to the complete solution of the system (5.1) provided $\psi_{2}\left(\xi_{1}, \xi_{2}\right) \neq 0$ or $\chi_{2}\left(\xi_{1}, \xi_{2}\right) \neq 0$. Using a computer algebra system, the polynomials $R_{1}$ and $R_{2}$ can be factorized as

$$
R_{1}\left(\xi_{1}\right)=\nu_{1}\left(\xi_{1}\right)^{2} Q\left(\xi_{1}\right), \quad R_{2}\left(\xi_{1}\right)=-\nu_{2}\left(\xi_{1}\right)^{2} Q\left(\xi_{1}\right),
$$

where $\nu_{i}$ form a basis of the elimination ideals

$$
\left\langle\nu_{1}\right\rangle=\left\langle\psi_{1}, \psi_{2}\right\rangle \cap \mathbb{R}\left[\xi_{1}\right], \quad \nu_{1}\left(\xi_{1}\right)=4 \xi_{1}^{5}+10 c_{3} \xi_{1}^{4}+\left(60 c_{3}^{2}-40 c_{4}\right) \xi_{1}^{3}+\ldots,
$$

and

$$
\left\langle\nu_{2}\right\rangle=\left\langle\chi_{1}, \chi_{2}\right\rangle \cap \mathbb{R}\left[\xi_{1}\right], \quad \nu_{2}\left(\xi_{1}\right)=\left(5 c_{3}^{2}-4 c_{4}\right) \xi_{1}^{12}+\ldots
$$

and $Q$ has the form as written in Lemma 5.1. If $\nu_{1}$ and $\nu_{2}$ have no common divisors, they are obviously extraneous factors in the equation (5.6), and

$$
\mathcal{V}\left(\mathcal{I}_{2}\right) \subseteq \mathcal{V}(Q) .
$$

If $\nu_{1}\left(\xi_{1}\right)=0, \nu_{2}\left(\xi_{1}\right)=0$ for some $\xi_{1} \in \mathbb{C}$, then $\operatorname{Res}\left(\nu_{1}\left(\xi_{1}\right), \nu_{2}\left(\xi_{1}\right) ; \xi_{1}\right)=0$ gives a tremendous, but polynomial relation between the constants $c_{i}$ that has to be satisfied. So the measure of the set of constants

$$
\left\{\left(c_{3}, c_{4}, \ldots, c_{9}\right) \in \mathbb{R}^{7} ; \nu_{1}\left(\xi_{1}\right)=0, \nu_{2}\left(\xi_{1}\right)=0\right\}
$$


is zero, and one may extend (5.7) to all possible constants by the continuity. Note that the inclusion (5.7) shows also that if $\xi_{1}=\xi_{1}\left(c_{3}, c_{4}, \ldots, c_{9}\right) \in \mathcal{V}\left(\mathcal{I}_{2}\right)$ grows unboundedly or decreases from infinity as a function of the constants $c_{i}$, then so must the corresponding $\xi_{1} \in \mathcal{V}(Q)$. So the difference of the number of solutions

$$
\# \mathcal{V}(Q)-\# \mathcal{V}\left(\mathcal{I}_{2}\right) \geq 0
$$

is independent of the constants $c_{i}$. But for a particular choice of the constants

$$
c_{3}=1, c_{4}=\frac{1}{2}, c_{5}=-1, c_{6}=1, c_{7}=0, c_{8}=-\frac{1}{2}, c_{9}=1
$$

it is straightforward to verify that $\mathcal{V}\left(\mathcal{I}_{2}\right)$ is equal to $\mathcal{V}(Q)$ since $\# \mathcal{V}\left(\mathcal{I}_{2}\right)=\# \mathcal{V}(Q)=$ 25.

Thus only the roots of $Q$ have to be considered. If the degree of $Q$ is odd, then $Q$ has at least one real root $\xi_{1}$ of odd multiplicity, which can be extended to a real element of $\mathcal{V}(\mathcal{I})$. Since $\xi_{1} \in \mathbb{R}$ is of odd multiplicity, a perturbation $\mathcal{O}(h)$ preserves the existence of a real solution, and the system (5.1) has at least one real solution for all $h$ small enough.

There remains to verify that $Q \not \equiv 0$ is of odd degree. The first step is obvious from Lemma 5.1 since $Q$ is of degree 25 unless the leading term vanishes. In this case,

and further,

$$
c_{4}=\frac{5}{4} c_{3}^{2}, \quad Q\left(\xi_{1}\right)=-\frac{1953125}{1048576}\left(7 c_{3}^{3}-4 c_{5}\right)^{4} \xi_{1}^{23}+\ldots
$$

$$
c_{4}=\frac{5}{4} c_{3}^{2}, c_{5}=\frac{7}{4} c_{3}^{3}, \quad Q\left(\xi_{1}\right)=-\frac{1953125}{65536}\left(21 c_{3}^{4}-8 c_{6}\right)^{4} \xi_{1}^{19}+\ldots .
$$

The additional assumptions

$$
c_{6}=\frac{21}{8} c_{3}^{4}, c_{7}=\frac{33}{8} c_{3}^{5}, c_{8}=\frac{429}{64} c_{3}^{6}
$$

reduce the degree of $Q$ to 15,11 and 7 . Note that regardless of the particular constants $c_{i}$ the polynomial $Q$ remains to be odd. Finally, the choice $c_{9}=\frac{715}{64} c_{3}^{7}$ gives $Q \equiv 0$, and any $\xi_{1} \in \mathbb{R}$ is suitable. It is not difficult to guess where this particular data curve comes from. One can easily verify that in this case

$$
\sum_{k=2}^{9} c_{k} h^{k-2} x^{k}
$$

is the Taylor polynomial of the function

$$
y(x)=\frac{1}{2 c^{2}}-\frac{x}{c}-\frac{\sqrt{1-4 c x}}{2 c^{2}}, \quad c:=\frac{h}{2} c_{3} .
$$

A straightforward computation leads to one of the possible reparametrizations of $f$ :

$$
\boldsymbol{f}(z)=\left(\begin{array}{c}
c z-c^{3} z^{2} \\
c^{2} z^{2}
\end{array}\right), \quad z:=z(c):=\frac{1-\sqrt{1-4 c x}}{2 c^{2}} .
$$

Thus $\boldsymbol{f}$ is a quadratic parametric polynomial. This remains true also as $c_{3} \rightarrow$ 0 , since $y(x) \rightarrow x^{2}$ in this case. Of course, the quintic geometric interpolation reproduces the quadratic parametric polynomial. 


\section{REFERENCES}

[1] Eugene L. Allgower and Kurt Georg, Numerical continuation methods, Springer Series in Computational Mathematics, vol. 13, Springer-Verlag, Berlin, 1990, An introduction. MR.1059455 (92a:65165)

[2] Carl de Boor, Klaus Höllig, and Malcolm Sabin, High accuracy geometric Hermite interpolation, Comput. Aided Geom. Design 4 (1987), no. 4, 269-278. MR937366 (90b:65014)

[3] Yu Yu Feng and Jernej Kozak, On spline interpolation of space data, Mathematical methods for curves and surfaces, II (Lillehammer, 1997), Innov. Appl. Math., Vanderbilt Univ. Press, Nashville, TN, 1998, pp. 167-174. MR.1640549 (99e:41024)

[4] K. Höllig and J. Koch, Geometric Hermite interpolation with maximal order and smoothness, Comput. Aided Geom. Design 13 (1996), no. 8, 681-695. MR1427357 (98m:65017)

[5] Jernej Kozak and Emil Žagar, On geometric interpolation by polynomial curves, SIAM J. Numer. Anal. 42 (2004), no. 3, 953-967 (electronic). MR2112788

[6] C. Krattenthaler, Advanced determinant calculus, Sém. Lothar. Combin. 42 (1999), Art. B42q, 67 pp. (electronic), The Andrews Festschrift (Maratea, 1998). MR1701596 (2002i:05013)

[7] Knut Mørken, Parametric interpolation by quadratic polynomials in the plane, Mathematical methods for curves and surfaces (Ulvik, 1994), Vanderbilt Univ. Press, Nashville, TN, 1995, pp. 385-402. MR.1356983 (96h:65023)

[8] Knut Mørken and Karl Scherer, A general framework for high-accuracy parametric interpolation, Math. Comp. 66 (1997), no. 217, 237-260. MR1372007 (97e:65026)

[9] Abedallah Rababah, High order approximation method for curves, Comput. Aided Geom. Design 12 (1995), no. 1, 89-102. MR:1311120 (95m:65030)

[10] Robert Schaback, Interpolation with piecewise quadratic visually $C^{2}$ Bézier polynomials, Comput. Aided Geom. Design 6 (1989), no. 3, 219-233. MR1019424 (90j:65020)

[11] Karl Scherer, Parametric polynomial curves of local approximation order 8, Curve and Surface Fitting (Saint Malo, 1999), Vanderbilt Univ. Press, Nashville, TN, 2000, pp. 375-384.

Institute of Mathematics, Physics and Mechanics, Jadranska 19, SI-1000 Ljubljana, SLOVENIA

E-mail address: gasper.jaklic@fmf.uni-lj.si

Department of Mathematics and Institute of Mathematics, Physics and Mechanics, Jadranska 19, SI-1000 LuUbluana, Slovenia

E-mail address: jernej.kozak@fmf.uni-lj.si

Institute of Mathematics, Physics and Mechanics, Jadranska 19, SI-1000 Luubljana, SLOVEnia

E-mail address: marjetka.krajnc@fmf.uni-lj.si

Department of Mathematics and Institute of Mathematics, Physics and Mechanics, Jadranska 19, SI-1000 LuUbluana, Slovenia

E-mail address: emil.zagar@fmf.uni-lj.si 